\title{
Confronting the Inert Doublet Model with results from Run 1 of the LHC
}

\section{Sengupta*†}

Laboratoire de Physique Subatomique et de Cosmologie, Université Grenoble-Alpes, CNRS/IN2P3, 53 Avenue des Martyrs, F-38026 Grenoble, France

E-mail: dipan.sengupta@lpsc.in2p3.fr

The Inert Doublet Model (IDM) is a simple extension of the Standard Model (SM) that aims to address the naturalness problem, electroweak baryogenesis and accommodate a viable dark matter (DM) candidate, along with a rich phenomenlogy in terms of collider signatures. In this note, we address the constraints on the IDM from dilepton searches performed at LHC run-1.

18th International Conference From the Planck Scale to the Electroweak Scale 25-29 May 2015

Ioannina, Greece

\footnotetext{
*Speaker.

${ }^{\dagger}$ In collaboration with : G. Belanger, B. Dumont, A. Goudelis, B. Herrmann and S.Kraml
} 


\section{Introduction}

The IDM, a simple extension of the two Higgs doublet model (2HDM) by an additional $\mathbb{Z}_{2}$ symmetry, has been studied for almost 4 decades in various contexts. Initially introduced as a model for electroweak symmetry breaking [1], the model has subsequently been found useful to address a whole array of issues, including improved naturalness [2], baryogenesis[3], generation of neutrino masses via see-saw mechanism [4], and importantly a viable dark matter candidate predicting the correct relic density of the universe [5-7]. The IDM provides a rich phenomenology that has been previously studied both in the context of LHC signatures [8-10], as well as astrophysical data on direct and indirect detection of dark matter [11-14]. Furthermore, theoretical constraints like vacuum stability, perturbativity, as well as constraints originating from electroweak precision data and dark matter have also been studied in some detail [15-17]. The discovery of the $125 \mathrm{GeV}$ Higgs boson $[18,19]$ has constrained the the IDM parameter space significantly. However, a study of constraints coming from the direct searches performed during LHC run-1 has not been conducted. In this work [20], we analyse the constraints on the IDM parameter space from the dilepton + missing energy $\left(E_{T}^{m i s s}\right)$ searches performed at LHC run-1. We recast two publicly available ATLAS analyses for the Electroweakino search [21] and invisible Higgs [22] in the MadAnalysis5 [23, 24] framework, now available on the public analysis database [25], and reinterpret them to constrain the IDM parameter space.

\section{IDM parameter space}

The IDM is an extension of the SM by the addition of a second SU(2) doublet scalar $\Phi$, being odd under the additional $\mathbb{Z}_{2}$ symmetry. The two doublets can be written as,

$$
H=\left(\begin{array}{c}
G^{+} \\
\frac{1}{\sqrt{2}}\left(v+h+i G^{0}\right)
\end{array}\right), \Phi=\left(\begin{array}{c}
H^{+} \\
\frac{1}{\sqrt{2}}\left(H^{0}+i A^{0}\right)
\end{array}\right) .
$$

The SM like Higgs boson is represented by h, while $H^{0}$ and $A^{0}$ denote the additional CP even neutral and CP odd neutral scalars respectively. Additionally it contains a pair charged scalars $\left(H^{ \pm}\right) . G^{0}$ and $G^{+}$represent the neutral and the charged goldstone bosons respectively. With these field definitions, the scalar part of the potential has the form,

$$
\begin{aligned}
V_{0} & =\mu_{1}^{2}|H|^{2}+\mu_{2}^{2}|\Phi|^{2}+\lambda_{1}|H|^{4}+\lambda_{2}|\Phi|^{4} \\
& +\lambda_{3}|H|^{2}|\Phi|^{2}+\lambda_{4}\left|H^{\dagger} \Phi\right|^{2}+\frac{\lambda_{5}}{2}\left[\left(H^{\dagger} \Phi\right)^{2}+\text { h.c. }\right] .
\end{aligned}
$$

The physical masses for the particles, given in terms of the Largrangian parameters read,

$$
\begin{aligned}
m_{h}^{2} & =\mu_{1}^{2}+3 \lambda_{1} v^{2}, \\
m_{H^{0}}^{2} & =\mu_{2}^{2}+\lambda_{L} v^{2}, \\
m_{A^{0}}^{2} & =\mu_{2}^{2}+\lambda_{S} v^{2}, \\
m_{H^{ \pm}}^{2} & =\mu_{2}^{2}+\frac{1}{2} \lambda_{3} v^{2},
\end{aligned}
$$


with the couplings $\lambda_{L, S}$ defined as,

$$
\lambda_{L, S}=\frac{1}{2}\left(\lambda_{3}+\lambda_{4} \pm \lambda_{5}\right)
$$

The $\mathbb{Z}_{2}$, symmetry ensures that the doublets $\mathrm{H}$ and $\Phi$ do not mix. A further consequence is that the lightest $\mathbb{Z}_{2}$ odd particle (LOP) $\left(H^{0}\right.$ or $\left.A^{0}\right)$, can be a candidate for a dark matter particle.

The following constraints are imposed on the IDM parameter space in this work,

1. The first set of constraints arises from theoretical requirements like stability of the electroweak vacuum, validity of perturbativity and unitarity of the model $[15,16]$. These three requirements are assumed to be valid up to $10 \mathrm{TeV}$.

2. The next set of constraints imposed are on the contributions of the IDM to the oblique parameters $S, T, U$. We use $3 \sigma$ ranges as described in [26].

3. Furthermore we impose LEP constraints from the neutralino and chargino searches [27]. We require (assuming $\left.m_{H^{0}}<m_{A^{0}}\right) m_{A^{0}} \gtrsim 100 \mathrm{GeV}$ and $m_{H^{ \pm}} \gtrsim m_{W}$.

4. For $m_{H^{0}} \leq m_{h} / 2$, (without the loss of generality) we must ensure that the constraints arising from the decay modes of the SM Higgs are in accordance to the latest experimental values. Assuming that the Higgs has SM-like couplings, the invisible branching ratio of the Higgs to two $H^{0}$ particles is constrained to $\mathrm{BR}(h \rightarrow$ inv. $)<0.12$ at $95 \%$ confidence level (CL) [28] (see also [29-32]). In this work, we work in the limit of $\lambda_{L} \rightarrow 0$, where constraints arising from invisible Higgs decays vanish.

Finally we assess the dark matter constraints on the IDM. If the IDM is considered as a viable dark matter model, it can satisfy the observed DM relic density [33] in three regions: the low mass regime, $m_{H^{0}}<m_{W}$, where $\lambda_{L}$, the coupling relating the two $H^{0}$ particles to a Higgs boson, and the mass difference $m_{h} / 2-m_{H^{0}}$ plays a crucial role ${ }^{1}$; the intermediate mass region $m_{W}<m_{H^{0}} \lesssim 115$ $\mathrm{GeV}$, where the relic density is governed by $m_{H^{0}}$ and $\lambda_{L}$; and finally the high mass region where all parameters of the scalar potential except $\lambda_{2}$ drastically affect the DM relic abundance. In this work the high mass region is not of interest. In the limit of $\lambda_{L} \rightarrow 0$, constraints arising from invisible Higgs decays vanish ${ }^{2}$. Furthermore, XENON100 [34] has already eliminated the entire low-tointermediate mass regime, where the IDM can satisfy the observed relic density according to the freeze out mechanism. Thus with the exception of a highly fine tuned Higgs funnel region, the $\lambda_{L} \rightarrow 0$ regime leads to a DM overabundance [17]. However one can always argue that the IDM can be treated as a model with interesting collider phenomenology without strictly imposing a DM constraint. In view of this we assess the constraints on the IDM from the LHC dilepton searches.

\section{LHC constraints on the IDM}

To assess the constraints on the IDM we considered the dilepton and the trilepton channels as the primary targets because of lower backgrounds and a cleaner signature. However the trilepton

\footnotetext{
${ }^{1}$ the exact difference between $m_{H^{0}}$ and $m_{W}$ also plays a role when the former is larger than $\sim 70 \mathrm{GeV}$

${ }^{2}$ The exact constraint is $\lambda_{L} \leq 6 \times 10^{-3}$, above which the entire low mass region is ruled out by $h \rightarrow i n v$.
} 
channel suffers significantly due to lower cross sections. Hence we consider the dilepton channel in this work.

The following four channels yielding a dilepton $+E_{T}^{\text {miss }}$ final state were considered in this work,

$$
\begin{aligned}
q \bar{q} & \rightarrow Z \rightarrow A^{0} H^{0} \rightarrow Z^{(*)} H^{0} H^{0} \rightarrow \ell^{+} \ell^{-} H^{0} H^{0}, \\
q \bar{q} & \rightarrow Z \rightarrow H^{ \pm} H^{\mp} \rightarrow W^{ \pm(*)} H^{0} W^{\mp(*)} H^{0} \\
& \rightarrow v \ell^{+} H^{0} v \ell^{-} H^{0} \\
q \bar{q} & \rightarrow Z \rightarrow Z h^{(*)} \rightarrow \ell^{+} \ell^{-} H^{0} H^{0} \\
q \bar{q} & \rightarrow Z \rightarrow Z H^{0} H^{0} \rightarrow \ell^{+} \ell^{-} H^{0} H^{0} .
\end{aligned}
$$

Although no dedicated search for the IDM has been performed at the LHC, ATLAS and CMS collaborations have performed studies with the same final state in the context of SUSY and invisible Higgs search [21]. These searches can be therefore be reinterpreted to constrain the IDM parameter space. To this end, we pick up two ATLAS searches, and recast them in the framework of MAdAnAlysis $5[23,24]$. The first of these is the ATLAS opposite side dilepton $+E_{T}^{\text {miss }}$ performed for the search of electroweakinos and sleptons [21].The $\ell^{+} \ell^{-}+E_{T}^{\text {miss }}$ signature can arise from chargino-pair production followed by $\tilde{\chi}^{ \pm} \rightarrow W^{ \pm(*)} \tilde{\chi}_{1}^{0}$ or $\tilde{\chi}^{ \pm} \rightarrow \ell^{ \pm} \tilde{v} / \tilde{v} \ell^{ \pm}$decays, or sleptonpair production followed by $\tilde{\ell}^{ \pm} \rightarrow \ell^{ \pm} \tilde{\chi}_{1}^{0}$ decays. For this analyses [21] the signal regions (SR) rely on purely leptonic final state. The analyses requires a veto on the $\mathrm{Z}$ boson to reduce the $\mathrm{ZZ}$ background, with $\left|m_{\ell \ell}-m_{Z}\right|>10 \mathrm{GeV}$. The $\ell^{+} \ell^{-}+E_{T}^{\text {miss }}$ signature corresponding to the simplified model $\tilde{\chi}^{+} \tilde{\chi}^{-} \rightarrow W^{+}\left(\rightarrow \ell^{+} v\right) \tilde{\chi}_{1}^{0} W^{-}\left(\rightarrow \ell^{-} v\right) \tilde{\chi}_{1}^{0}$ considered in the analyses can be directly mapped the IDM channel described in Eq. 3.2.

The second ATLAS search of interest is the search for invisible decays of a Higgs boson produced in association with a $Z$ boson [22]. This search is complementary to the electroweakino search in accepting the reconstructed $\mathrm{Z}$ boson from the $\ell^{+} \ell^{-}$pair, the specific requirement being $\left|m_{\ell \ell}-m_{Z}\right|<15 \mathrm{GeV}$. This process is a direct analogue of Eq. 3.3 and 3.4. Additionally, a dark matter search with $\ell^{+} \ell^{-}+E_{T}^{\text {miss }}$ [35] final state was also performed. However the analyses requires a large missing energy criteria which wipes out the entire signal.

As noted earlier, these two ATLAS analyses are recasted using the MADANALYSIS 5 [23, 24] framework. While the SUSY search [21] was already available in the Public Analysis Database [25] as the recast code [36], the invisible Higgs search [22] was implemented and validated for this work and is now available at [37]. For the recast, signal samples were generated with Madgraph5 [38] using the model implemented using Feynrules available in [17], with the widths calculated by Calchep [39, 40]. The parton-level events were passed on to Pythia 6.4 [41] for showering and hadronization. The 'MA5tune' version of DeLPhes 3 [42] (see Section 2.2 of [25]) was used for detector simulation. The cutflows were obtained with the recast codes described in [36, 37]. To obtain a statistical interpretation, we used the module exclusion_CLs.py [25]. With the given number of signal, observed and expected background events, along with the background uncertainty, exclusion_CLS.Py determines the most sensitive SR, the exclusion confidence level using the $\mathrm{CL}_{s}$ prescription, and the nominal cross section $\sigma_{95}$ that is excluded at $95 \% \mathrm{CL} .{ }^{3}$

\footnotetext{
${ }^{3}$ Note that we do not simulate the backgrounds but take the background numbers and uncertainties directly from the experimental publications [21] and [22].
} 
With the imposition of $\lambda_{L}=0$, and noting that $\lambda_{2}$ is irrelevant for all observables at treelevel, we are left with $m_{A^{0}}$ and $m_{H^{0}}$ to scan over. For $m_{H^{ \pm}}$, we choose two representative values: $m_{H^{ \pm}}=85 \mathrm{GeV}$, which is the lower allowed limit by LEP, and $m_{H^{ \pm}}=150 \mathrm{GeV}$, which is significantly higher but still safely within the bounds imposed by the $T$ parameter, which limits the mass splitting between the inert scalar states (see also the analysis in [17]).

The result for the recasted analyses is presented in Fig. 1, where we show $\mu \equiv \sigma_{95} / \sigma_{\text {IDM }}$ in the form of temperature plots in the $\left(m_{A^{0}}, m_{H^{0}}\right)$ plane for the two chosen values of $m_{H^{ \pm}}$. In these plots $\sigma_{\text {IDM }}$ is the cross section predicted by the model while $\sigma_{95}$ is the cross section excluded at 95\% CL. Thus regions with $\mu \leq 1$ are excluded at 95\% CL.
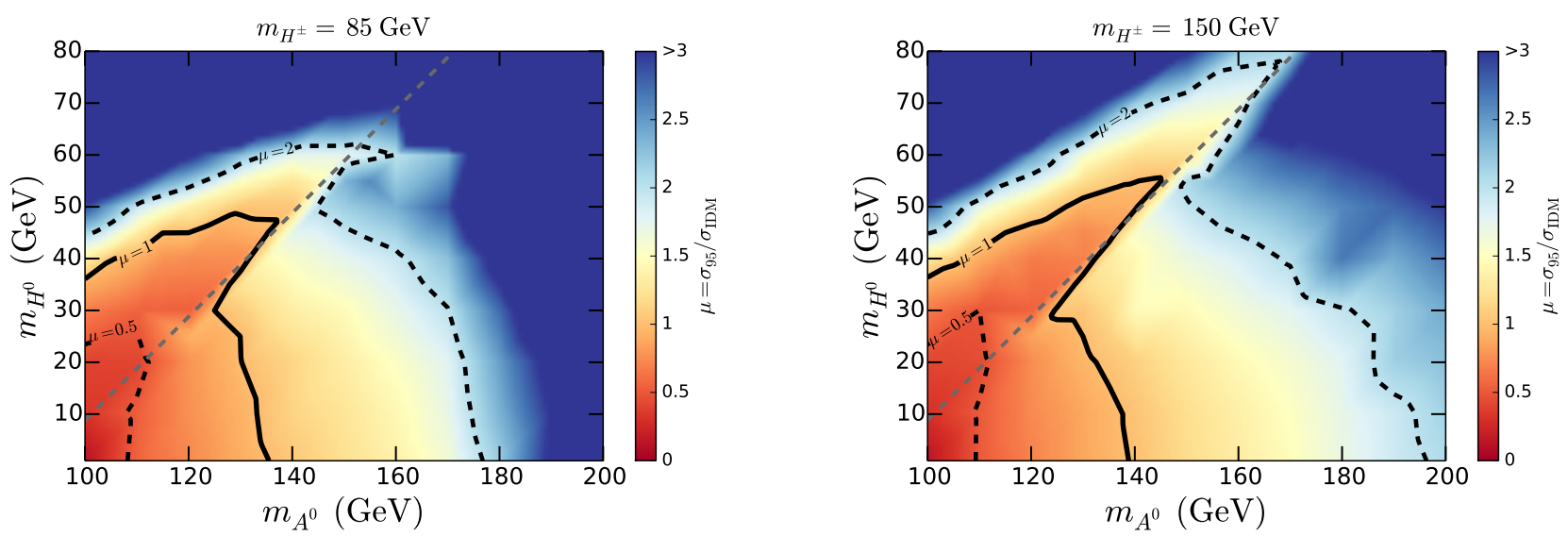

Figure 1: The ratio $\mu \equiv \sigma_{95} / \sigma_{\text {IDM }}$ in the $\left(m_{A^{0}}, m_{H^{0}}\right)$ plane for two representative values of the charged inert scalar mass, $m_{H^{ \pm}}=85 \mathrm{GeV}$ (upper panel) and $m_{H^{ \pm}}=150 \mathrm{GeV}$ (lower panel). The solid black lines are the 95\% CL exclusion contours, $\mu=1$. The dashed black lines are given for illustration and correspond to the $\mu=0.5$ and $\mu=2$ contours. The grey dashed lines indicate $m_{A^{0}}-m_{H^{0}}=m_{Z}$.

We observe that Run 1 ATLAS dilepton searches exclude inert scalar masses up to about $35 \mathrm{GeV}$ for pseudoscalar masses around $100 \mathrm{GeV}$ at $95 \% \mathrm{CL}$. The limits become stronger for larger $m_{A^{0}}$, with $m_{H^{0}} \approx 45$ (55) GeV ruled out for $m_{A^{0}} \approx 140$ (145) GeV and $m_{H^{ \pm}}=85$ (150) GeV. For massless $H^{0}, A^{0}$ masses up to about $135-140 \mathrm{GeV}$ are excluded ( $m_{H^{0}}$ and $m_{A^{0}}$ are generally interchangeable here). It can be observed that the constraints are slightly stronger for heavier $m_{H^{ \pm}}$. This is because of sub-leading contributions from Eq. 3.2 and from $q \bar{q} \rightarrow W^{ \pm} \rightarrow A H^{ \pm} \rightarrow$ $Z^{(*)} H W^{ \pm(*)} H$ with a missed lepton. Although the cross section is much larger for $m_{H^{ \pm}}=85 \mathrm{GeV}$ as compared to $m_{H^{ \pm}}=150 \mathrm{GeV}$, the resulting leptons are much softer and generally do not pass the signal requirements.

It can also be observed the limits on $m_{H^{0}}$ become stronger for larger $A^{0}$ masses. This is because the leptons originating from the $A^{0} \rightarrow Z^{(*)} H^{0}$ decay are harder with more available phase space and thus pass the signal selection cuts more easily. On the other hand for smaller mass splittings between $H^{0}$ and $A^{0}$, the produced dileptons are much softer.

On the other hand, when $m_{A^{0}}-m_{H^{0}} \geq m_{Z}$, the $Z$ boson is on-shell, and hence the $Z$ veto in the SUSY analysis eliminates most of the signal. In this region the invisible Higgs analsyses, $Z h \rightarrow \ell^{+} \ell^{-}+E_{T}^{\text {miss }}$ provides the stronger limit. Although we use $m_{H^{ \pm}}=150 \mathrm{GeV}$ in this work, the mass of the charged scalar has a negligible effect in the final exclusion. It can be noticed that there 
is a small overlap region at low $H^{0}$ mass, $m_{H^{0}} \lesssim 25 \mathrm{GeV}$, where the SUSY search constrain a small region despite the $Z$ boson from the $A^{0}$ decay being on-shell. This comes from the significantly large tail of the $m_{\ell \ell}$ distribution extending below $m_{\ell \ell}=m_{Z}-10 \mathrm{GeV}$, where some signal events are picked up in the SUSY SR $W W b$ (this tail was already noticed in [9]).

Finally a discussion on the prospects of the $13 \mathrm{TeV}$ Run is worthwhile. With the minimal assumption that one can naïvely rescale signal and background numbers (see, e.g., [43]), (assuming that the acceptance $\times$ efficiency values remain the same) indicates that at $13 \mathrm{TeV}$ with an integrated luminosity of $100 \mathrm{fb}^{-1}$, the $95 \% \mathrm{CL}$ reach can be extended up to $\mu \approx 1.2(1.6)$ above (below) the dashed grey line in Figure 1 . This reach improves to $\mu \approx 2.1$ (2.7) with a luminosity of $300 \mathrm{fb}^{-1}$, hence covering a major part of the $\left(m_{A^{0}}, m_{H^{0}}\right)$ plane shown in Figure 1. This reach, however depends on a number of factors including detector performance affecting the signal and background estimation, the actual change in kinematics at an increased energy. Furthermore, we advocate that a dedicated search should be devised to investigate this model, with an analysis targeted specifically for $p p \rightarrow A^{0} H^{0} \rightarrow Z^{(*)} H^{0} H^{0}$ (or alternatively $p p \rightarrow \tilde{\chi}_{2}^{0} \tilde{\chi}_{1}^{0} \rightarrow Z^{(*)} \tilde{\chi}_{1}^{0} \tilde{\chi}_{1}^{0}$ ). Variables like angular separation between leptons can be helpful in this respect.

\section{Conclusion}

In this work we addressed the constraints on the Inert Doublet Model coming from dilepton + $E_{T}^{\text {miss }}$ searches at LHC. We conclude that the Run 1 of LHC provide significant constraints on the IDM parameter space, extending them beyond the LEP, and complementary to cosmological and astrophysical constraints. The $95 \% \mathrm{CL}$ limits derived from the dilepton $+E_{T}^{\text {miss }}$ Higgs and SUSY analyses exclude inert scalar masses of up to about $55 \mathrm{GeV}$ in the best cases. It should be possible to push these constraints beyond $m_{h} / 2 \approx 62.5 \mathrm{GeV}$ at Run 2 of the LHC, thus enabling to probe the Higgs funnel region of the IDM, a regime. However a dedicated experimental analysis for the IDM at $13 \mathrm{TeV}$ would be highly desirable, as it would enable to constrain the parameter space beyond the recasted analysis with optimized cuts.

\section{Acknowledgements}

This work was supported in part by the ANR project DMASTROLHC, ANR-12-BS05-0006, the "Investissements d'avenir, Labex ENIGMASS", and by the IBS under Project Code IBS-R018D1.

\section{References}

[1] N. G. Deshpande and E. Ma, "Pattern of Symmetry Breaking with Two Higgs Doublets," Phys. Rev. D 18 (1978) 2574.

[2] R. Barbieri, L. J. Hall, and V. S. Rychkov, "Improved naturalness with a heavy Higgs: An Alternative road to LHC physics," Phys. Rev. D 74 (2006) 015007 , arXiv:hep-ph/0603188 [hep-ph]. 
[3] E. Ma, "Common origin of neutrino mass, dark matter, and baryogenesis," Mod. Phys. Lett. A21 (2006) 1777-1782, arXiv:hep-ph/0605180 [hep-ph].

[4] E. Ma, "Verifiable radiative seesaw mechanism of neutrino mass and dark matter," Phys. Rev. D73 (2006) 077301, arXiv: hep-ph/ 0601225 [hep-ph] .

[5] L. Lopez Honorez, E. Nezri, J. F. Oliver, and M. H. Tytgat, "The Inert Doublet Model: An Archetype for Dark Matter," JCAP 0702 (2007) 028, arXiv: hep-ph / 0612275 [hep-ph] .

[6] T. Hambye and M. H. Tytgat, "Electroweak symmetry breaking induced by dark matter," Phys. Lett. B 659 (2008) 651-655, arXiv:0 0707.0633 [hep-ph] .

[7] L. Lopez Honorez and C. E. Yaguna, “A new viable region of the inert doublet model," JCAP 1101 (2011) 002, arXiv:1011.1411 [hep-ph] .

[8] Q.-H. Cao, E. Ma, and G. Rajasekaran, "Observing the Dark Scalar Doublet and its Impact on the Standard-Model Higgs Boson at Colliders," Phys. Rev. D 76 (2007) 095011, arXiv:0708.2939 [hep-ph].

[9] E. Dolle, X. Miao, S. Su, and B. Thomas, "Dilepton Signals in the Inert Doublet Model," Phys. Rev. D 81 (2010) 035003, arXiv:0909.3094 [hep-ph] .

[10] A. Arhrib, R. Benbrik, and N. Gaur, " $H \rightarrow \gamma \gamma$ in Inert Higgs Doublet Model," Phys. Rev. $\sim D$ 85 (2012) 095021, arXiv:1201.2644 [hep-ph].

[11] M. Gustafsson, E. Lundström, L. Bergström, and J. Edsjö, "Significant Gamma Lines from Inert Higgs Dark Matter," Phys. Rev. Lett. 99 (2007) 041301, arXiv:astro-ph/0703512 [astro-ph].

[12] P. Agrawal, E. M. Dolle, and C. A. Krenke, "Signals of Inert Doublet Dark Matter in Neutrino Telescopes," Phys. Rev. D 79 (2009) 015015, arXiv:0 811.1798 [hep-ph] .

[13] S. Andreas, M. H. Tytgat, and Q. Swillens, "Neutrinos from Inert Doublet Dark Matter," JCAP 0904 (2009) 004, arXiv: 0901.1750 [hep-ph ].

[14] M. Gustafsson, S. Rydbeck, L. Lopez-Honorez, and E. Lundstrom, "Status of the Inert Doublet Model and the Role of multileptons at the LHC," Phys.Rev. D86 (2012) 075019, arXiv:1206.6316 [hep-ph].

[15] N. Khan and S. Rakshit, "Constraints on inert dark matter from metastability of electroweak vacuum," arXiv:1503.03085 [hep-ph].

[16] B. Swiezewska, "Inert scalars and vacuum metastability around the electroweak scale," arXiv:1503.07078 [hep-ph]. 
[17] A. Goudelis, B. Herrmann, and O. Stal, "Dark matter in the Inert Doublet Model after the discovery of a Higgs-like boson at the LHC," JHEP 1309 (2013) 106, arXiv: 1303.3010 [hep-ph].

[18] ATLAS Collaboration Collaboration, G. Aad et al., "Observation of a new particle in the search for the Standard Model Higgs boson with the ATLAS detector at the LHC," Phys. Lett. B 716 (2012) 1-29, arXiv:1207.7214 [hep-ex].

[19] CMS Collaboration Collaboration, S. Chatrchyan et al., "Observation of a new boson at a mass of $125 \mathrm{GeV}$ with the CMS experiment at the LHC," Phys. Lett. B 716 (2012) 30-61, arXiv:1207.7235 [hep-ex].

[20] G. Belanger, B. Dumont, A. Goudelis, B. Herrmann, S. Kraml, and D. Sengupta, "Dilepton constraints in the Inert Doublet Model from Run 1 of the LHC," Phys. Rev. D91 no. 11, (2015) 115011, arXiv:1503.07367 [hep-ph] .

[21] ATLAS Collaboration Collaboration, G. Aad et al., "Search for direct production of charginos, neutralinos and sleptons in final states with two leptons and missing transverse momentum in $p p$ collisions at $\sqrt{s}=8 \mathrm{TeV}$ with the ATLAS detector," JHEP 1405 (2014) 071, arXiv:1403.5294 [hep-ex].

[22] ATLAS Collaboration Collaboration, G. Aad et al., "Search for Invisible Decays of a Higgs Boson Produced in Association with a Z Boson in ATLAS," Phys.Rev.Lett. 112 (2014) 201802, arXiv:1402.3244 [hep-ex].

[23] E. Conte, B. Dumont, B. Fuks, and C. Wymant, "Designing and recasting LHC analyses with MadAnalysis 5," Eur.Phys.J. C74 no. 10, (2014) 3103, arXiv:1405.3982 [hep-ph] .

[24] E. Conte, B. Fuks, and G. Serret, "MadAnalysis 5, A User-Friendly Framework for Collider Phenomenology," Comput.Phys.Commun. 184 (2013) 222-256, arXiv:1206.1599 [hep-ph] .

[25] B. Dumont, B. Fuks, S. Kraml, et al., "Toward a public analysis database for LHC new physics searches using MADANALYSIS 5," Eur.Phys.J. C75 no. 2, (2015) 56, arXiv:1407.3278 [hep-ph].

[26] M. Baak, M. Goebel, J. Haller, A. Hoecker, D. Ludwig, et al., "Updated Status of the Global Electroweak Fit and Constraints on New Physics,” Eur.Phys.J. C72 (2012) 2003, arXiv:1107.0975 [hep-ph].

[27] E. Lundström, M. Gustafsson, and J. Edsjö, “The Inert Doublet Model and LEP II Limits," Phys. Rev. D 79 (2009) 035013, arXiv:0810.3924 [hep-ph] .

[28] J. Bernon, B. Dumont, and S. Kraml, "Status of Higgs couplings after run 1 of the LHC," Phys.Rev. D90 no. 7, (2014) 071301, arXiv:1409.1588 [hep-ph] .

[29] G. Belanger, B. Dumont, U. Ellwanger, J. Gunion, and S. Kraml, "Status of invisible Higgs decays," Phys.Lett. B723 (2013) 340-347, arXiv:1302.5694 [hep-ph] . 
[30] A. Falkowski, F. Riva, and A. Urbano, "Higgs at last," JHEP 1311 (2013) 111, arXiv:1303.1812 [hep-ph].

[31] G. Belanger, B. Dumont, U. Ellwanger, J. Gunion, and S. Kraml, "Global fit to Higgs signal strengths and couplings and implications for extended Higgs sectors," Phys.Rev. D88 (2013) 075008, arXiv:1306.2941 [hep-ph] .

[32] P. Bechtle, S. Heinemeyer, O. Stål, T. Stefaniak, and G. Weiglein, "Probing the Standard Model with Higgs signal rates from the Tevatron, the LHC and a future ILC," JHEP 1411 (2014) 039, arXiv:1403.1582 [hep-ph] .

[33] Planck Collaboration, P. Ade et al., "Planck 2013 results. XVI. Cosmological parameters," Astron.Astrophys. 571 (2014) A16, arXiv:1303.5076 [astro-ph.CO].

[34] XENON100 Collaboration Collaboration, E. Aprile et al., "Dark Matter Results from 225 Live Days of XENON100 Data," Phys.Rev.Lett. 109 (2012) 181301, arXiv:1207. 5988 [astro-ph.CO].

[35] ATLAS Collaboration Collaboration, G. Aad et al., "Search for dark matter in events with a $\mathrm{Z}$ boson and missing transverse momentum in pp collisions at $\sqrt{s}=8 \mathrm{TeV}$ with the ATLAS detector," Phys.Rev. D90 no. 1, (2014) 012004, arXiv:1404.0051 [hep-ex] .

[36] B. Dumont, "MadAnalysis 5 implementation of ATLAS-SUSY-2013-11: di-leptons plus MET,".

[37] B. Dumont, "MadAnalysis 5 implementation of ATLAS-HIGG-2013-03,".

[38] J. Alwall, M. Herquet, F. Maltoni, O. Mattelaer, and T. Stelzer, "MadGraph 5 : Going Beyond," JHEP 1106 (2011) 128, arXiv:1106.0522 [hep-ph] .

[39] A. Pukhov, "CalcHEP 2.3: MSSM, structure functions, event generation, batchs, and generation of matrix elements for other packages," arXiv: hep-ph/0412191 [hep-ph].

[40] A. Belyaev, N. D. Christensen, and A. Pukhov, "CalcHEP 3.4 for collider physics within and beyond the Standard Model,” Comput.Phys.Commun. 184 (2013) 1729-1769, arXiv:1207.6082 [hep-ph].

[41] T. Sjostrand, S. Mrenna, and P. Z. Skands, "PYTHIA 6.4 Physics and Manual," JHEP 0605 (2006) 026, arXiv:hep-ph/0603175 [hep-ph] .

[42] DELPHES 3 Collaboration, J. de Favereau et al., "DELPHES 3, A modular framework for fast simulation of a generic collider experiment," JHEP 1402 (2014) 057, arXiv:1307.6346 [hep-ex].

[43] A. Bharucha, S. Heinemeyer, and F. von der Pahlen, "Direct Chargino-Neutralino Production at the LHC: Interpreting the Exclusion Limits in the Complex MSSM," Eur.Phys.J. C73 no. 11, (2013) 2629, arXiv:1307.4237. 